\title{
A Pilot Study of Abnormal Growth in Autism Spectrum Disorders and Other Childhood Psychiatric Disorders
}

\author{
Nanda N. J. Rommelse $\cdot$ Cindy T. R. Peters $\cdot$ Iris J. Oosterling $•$ \\ Janne C. Visser • Daniëlle Bons · Daphne J. van Steijn • \\ Jos Draaisma $\cdot$ Rutger-Jan van der Gaag $\cdot$ Jan. K. Buitelaar
}

Published online: 29 April 2010

(C) The Author(s) 2010. This article is published with open access at Springerlink.com

\begin{abstract}
The aims of the current study were to examine whether early growth abnormalities are (a) comparable in autism spectrum disorders (ASD) and other childhood psychiatric disorders, and (b) specific to the brain or generalized to the whole body. Head circumference, height, and weight were measured during the first 19 months of life in 129 children with ASD and 59 children with nonASD psychiatric disorders. Both groups showed comparable abnormal patterns of growth compared to population norms, especially regarding height and head circumference in relation to height. Thus abnormal growth appears to be related to psychiatric disorders in general and is mainly expressed as an accelerated growth of height not matched by an increase in weight or head circumference.
\end{abstract}

Keywords Autism - Growth - Head circumference . Height · Weight · Endophenotype

N. N. J. Rommelse $(\varangle) \cdot$ C. T. R. Peters .

I. J. Oosterling · J. C. Visser - D. Bons .

D. J. van Steijn · R.-J. van der Gaag · Jan. K. Buitelaar

Karakter, Child and Adolescent Psychiatry University Center,

Nijmegen, The Netherlands

e-mail: n.lambregts-rommelse@psy.umcn.nl

C. T. R. Peters

e-mail: cindypeters18@hotmail.com

I. J. Oosterling

e-mail: i.oosterling@karakter.com

J. C. Visser

e-mail: j.visser@karakter.com

D. Bons

e-mail: d.bons@karakter.com

D. J. van Steijn

e-mail: d.vansteijn@karakter.com

R.-J. van der Gaag

e-mail: r.vandergaag@karakter.com

\section{Introduction}

Autism spectrum disorders (ASDs) are neurodevelopmental disorders characterized by an early onset of severe impairment in social interaction, abnormalities in communication, and restricted and stereotyped patterns of behavior and interests. Symptoms usually start before 3 years of age, and studies of children as young as 12 months of age demonstrate that certain behaviors are predictive of autism later in life, indicating that the origin of the disorder lies very early in development (Lawler et al. 2004). One of the earliest signs of abnormality in ASD is abnormal growth of the brain. MRI and postmortem studies have shown children with ASD to have cerebral, cerebellar, and limbic abnormalities (reviewed by Courchesne et al. 2004). The mean brain volume of children with ASD is greater than that of normal children during childhood, with

Jan. K. Buitelaar

e-mail: j.buitelaar@psy.umcn.nl

N. N. J. Rommelse · R.-J. van der Gaag · Jan. K. Buitelaar Department of Psychiatry, Radboud University Nijmegen Medical Centre, Reinier Postlaan 12, 6525 GC Nijmegen, The Netherlands

N. N. J. Rommelse - Jan. K. Buitelaar

Donders Institute for Brain, Cognition and Behaviour, Centre for Cognitive Neuroimaging, Radboud University Nijmegen, Nijmegen, The Netherlands

J. Draaisma

Department of Pediatrics, Radboud University Nijmegen

Medical Centre, Nijmegen, The Netherlands

e-mail: J.Draaisma@cukz.umcn.nl 
the difference in brain size peaking between 4 and 5 years of age (Courchesne et al. 2001, 2003; Hazlett et al. 2005; Sparks et al. 2002; reviewed in Redcay and Courchesne 2005). In addition, several studies have shown that some areas of the brain are enlarged whereas other are decreased in size (Carper et al. 2002; Courchesne 1997; Courchesne et al. 2001; Hazlett et al. 2005; Sparks et al. 2002; reviewed in Courchesne et al. 2004). However, these studies all involved children with autism who were older than 2 years, probably because of diagnostic uncertainty in children younger than this age (Wiggins et al. 2006; Zwaigenbaum et al. 2009). Diagnostic uncertainty is a major obstacle to understanding the earliest signs of abnormal brain development in these children.

A way to overcome this problem is to measure indices of brain development before the diagnosis is established. In most western countries, head circumference is measured several times during the first year of life in well-baby clinics, to monitor development, and head circumference is an accurate index of brain size in the first years of life (Bartholomeusz et al. 2002). In this way, abnormalities of brain development can be detected early in children later diagnosed with ASD. Most studies measuring head circumference data from birth to $\sim 3$ years of age reported, on average, an increased head circumference in autistic children (Dawson et al. 2007; Dementieva et al. 2005; Elder et al. 2008; Fukumoto et al. 2008; Gillberg and de Souza 2002; Hazlett et al. 2005; Lainhart et al. 1997, 2006; Mills et al. 2007; Webb et al. 2007), although two other studies reported a normal head circumference in autistic children of the same age (van Daalen et al. 2007; Torrey et al. 2004). However, it is probable that children with ASD have an atypical growth pattern (Amaral et al. 2008): at birth, children with ASD appear to have a normal or even decreased head circumference, followed by an increase in the rate of growth of head circumference from about 12 months of age (Amaral et al. 2008; Courchesne et al. 2003; Dissanayake et al. 2006; Mraz et al. 2007). Most research findings indicate that head growth abnormalities are present in a proportion of autistic children, both in terms of a larger head circumference and an atypical acceleration of growth.

Measurement of head circumference in relation to height may provide insight into these growth abnormalities, because the increase in head size may be associated with a general increase in growth in children with ASD. Some studies have documented that head circumference is relatively increased in comparison to height (Dawson et al. 2007; Fukumoto et al. 2008; Lainhart et al. 2006), whereas others have reported that head circumference is normal or decreased in relation to height (van Daalen et al. 2007; Mraz et al. 2007). Several studies have reported an increased height and/or weight in ASD (van Daalen et al.
2007; Dissanayake et al. 2006; Fukumoto et al. 2008; Lainhart et al. 1997; Mills et al. 2007; Mraz et al. 2007; Torrey et al. 2004; Webb et al. 2007; Whitely et al. 2004; Xiong et al. 2007), which suggests that growth in general is increased in ASD.

Because growth parameters are often found to be abnormal in ASD and are to a large part genetically determined (Carmichael and McGue 1995; Maes et al. 1997; Silventoinen et al. 2000), growth abnormalities have been proposed as candidate endophenotypes of ASD (Losh et al. 2008). Endophenotypes are subclinical behavioral, physiological, or neuropsychological markers of a disease that are suspected to be more strongly linked to certain genes than the clinical outcomes of the disease (Gottesman and Gould 2003). Endophenotypes may be particularly useful for exploring different pathways leading to a disorder, because homogeneous subgroups of patients sharing an endophenotypic dysfunction would be expected to share certain genetic features. Thus parameters of growth could be used to distinguish subtypes of ASD that are genetically more homogeneous. However, it has also been argued that endophenotypes should be disorder specific (Skuse 2001). In this case, the question arises whether or not growth abnormalities are specific to ASD or whether they are the non-specific expression of the biological abnormalities that are found in association with several psychiatric disorders (Skuse 2001). This has hardly been investigated. To the best of our knowledge, only one recent study has compared longitudinal growth parameters in children with ASD or developmental problems other than ASD (Webb et al. 2007). They reported that children with ASD had a larger average head circumference at 1-7 months and a larger average height at 1-4 months than children with developmental disabilities. At the age of 10 months, the children with ASD were larger than the developmentally disabled children, having a larger head and a greater height and weight. However, another study failed to find a difference in head circumference at birth between autistic children and children with attention-deficit/hyperactivity disorder (ADHD; Gillberg and de Souza 2002). These discrepant findings and the small sample sizes on which they are based (28 ASD children and 8 children with developmental disabilities in the Webb study) make it important to replicate these findings in a larger sample.

Therefore, the main aim of this study was to determine whether abnormal growth is predominantly associated with ASD or is a more universal finding in children with psychiatric disorders. Growth abnormalities are in no way uniquely associated with ASD, being also found in Canavan's and tuberous sclerosis. However, the extent to which growth abnormalities are present in other psychiatric disorders has hardly been investigated. We measured head circumference, which reflects brain volume in the first few 
years of life, and height and weight, to examine whether abnormal growth is specific to the brain/head or generalized to the whole body. Unlike previous studies, we had a relatively large sample (129 ASD and 59 psychiatric controls, PC) and measured the variables of interest several times $(N=9)$ during the first 1.5 years of life. We also took the effects of prematurity into account by analyzing the findings with and without premature children included.

\section{Method}

\section{Participants}

A total of 188 children with data on growth parameters during the first 1.5 years of life were included. The sample was a subsample of 251 children recruited to the DIANE study (Diagnosis and Intervention of Autism in the Netherlands; Oosterling et al. 2010). The DIANE project included children aged 1-3 years with a positive score $(\geq 3)$ on the ESAT questionnaire (Early Screening of Autistic Traits Questionnaire; Swinkels et al. 2006) and/or for whom there were major concerns regarding social communicative development. Exclusion criterion was a suspected non-ASD developmental disorder. More than $88 \%$ of our sample was diagnosed when older than 2 years, an age when reliable diagnosis is possible (Chawarska et al. 2007). In line with recommendations (Zwaigenbaum et al. 2009), the diagnostic protocol included: (a) psychiatric evaluation; (b) parent-child play observation; (c) the ADOS (Lord et al. 2001); (d) the ADI-R (Rutter et al. 2003); (e) IQ measured with the Mullen Scales of Early Learning (Mullen 1995) or with the Psychoeducational Profile Revised (for the latter scale, IQ was calculated as follows: (developmental age in months/chronological age in months) * 100; Schopler et al. 1990); and (f) language skills measured with the Dutch modification of the Reynell Developmental Language Scales (Van Eldik et al. 1995), the Schlichting Test for language production (1995), or the Dutch pre-verbal speech test (NNST; Zink and Lembrechts 2000), which measures pre-verbal skills such as imitation, social babbling, and use of simple gestures. The ADOS and ADI-R were administered by experienced clinical psychologists who met standard requirements for research reliability. Using the data provided by this extensive multidisciplinary diagnostic procedure, at least two experienced professionals, a child psychiatrist and a psychologist, made consensus best-estimate clinical diagnoses (BECD; American Psychiatric Association 2000). An ASD diagnosis was therefore not necessarily based on meeting ASD criteria on both the ADI-R and ADOS. The DSM-IV-TR (APA 2000) criteria, or if not applicable, the Diagnostic Classification of Mental Health and Developmental
Disorders of Infancy and Early Childhood: Revised edition (DC: $0-3 R ; 2005)$ criteria, were used to establish the final diagnosis; the child psychiatrist had final responsibility for the diagnosis.

In total, 129 children (109 male and 20 female) were diagnosed with ASD: 90 with autistic disorder, 37 with PDD-NOS, and 2 with Asperger's. Fifty-nine children (46 male and 13 female) with non-ASD psychiatric disorders (Psychiatric controls, PC) also participated: 39 had a psychiatric disorder other than ASD (such as ADHD, oppositional defiant disorder, communication disorder), 12 had a diagnosis according to the DC: 0-3R (2005; such as regulation problems), and 8 had mental retardation without a psychiatric problem. The relatively high number of nonASD cases was caused by the high sensitivity, yet relatively low specificity, of the 14-item screening instrument (ESAT) used for inclusion purposes (Swinkels et al. 2006). The ESAT has a high specificity to differentiate normal from abnormal development, but is less specific in distinguishing between ASD and other types of abnormal development, such as ADHD or mental retardation (Swinkels et al. 2006). The groups did not differ in terms of sex, duration of pregnancy, or number of premature births. However, the groups did differ in IQ, with relatively more children in the ASD group having a low IQ compared with children in the PC group. Most children (90\%) were of Dutch Caucasian descent; the remaining 10\% were of nonwestern ethnic descent.

\section{Instruments}

Head circumference, height, and weight were measured by trained health professionals at each visit to the well-baby clinic. Head circumference (maximum occipital-frontal head circumference) was measured with a plastic, nonstretchable measuring tape. Height was measured with the infants in supine position, fully extended, with their heels in contact with the baseboard. When they were older, height was measured with the infant standing erect against the wall. Infants up to age of 15 months were weighed naked, on calibrated baby scales.

\section{Procedures}

There were nine measuring moments $(0,1,2,3,4,5-6,7-$ 9, 10-13, 14-19 months). Head circumference was measured up to 1 year. Measurements were standardized for sex and age by converting the data into $z$-scores (measurements expressed in standard deviations from the population mean). The population mean was based on population norms from the database of the Netherlands Organization for Applied Scientific Research (Fredriks 
et al. 2000), and included data about head circumference, height, and weight from 14,500 boys and girls between the age of 0 and 20 years in 1996 and 1997. The proportional relationship between head circumference and height was calculated by subtracting the $z$-score of the height from the $z$-score of the head circumference for every child and for each age interval for which both scores were available (based on van Daalen et al. 2007 and Lainhart et al. 2006). A positive $z$-score indicated that the head was large relative to height; a negative $z$-score indicated that the head was small relative to height.

\section{Statistical Analyses}

From birth to 19 months, head circumference was measured seven times and height and weight nine times. All children had data for at least five of seven head circumference measurements and for at least seven of nine height and weight measurements. Missing data were replaced using expectation maximization (Tabachnick and Fidell 2001). A repeated-measures ANOVA was used with group as between subject factor (two groups: ASD and PC) and the different measurements as within subject factor. The effect of group was analyzed to examine structural group differences in the growth parameters. In addition, group by growth parameter interactions were tested, to examine whether or not the growth curves ran parallel for both groups. Lastly, the data were tested against a straight line through zero, which represented a growth curve completely in conformity with the normative data. If the growth curves did not pass through zero in a straight line, $z$-scores of individual measurements were analyzed in one-sample $t$-tests against a test value of zero. Abnormal growth acceleration was calculated based on Courchesne et al. (2003), with abnormal growth acceleration being defined as a change of $>2$ SD from birth to 14-19 months of age. We did the same for height, weight, and BMI. We defined abnormal growth acceleration for head circumference and head circumference minus height as a change of $>1.8 \mathrm{SD}$ from 1 to 10-13 months of age, because we did not have head circumference data from around the time of birth and at 14-19 months of age. Chi square tests were used to examine group differences in the number of children with microcephaly ( $\leq 2$ SD below population mean) and macrocephaly ( $\geq 2 \mathrm{SD}$ above population mean).

The data excluding children born at term or prematurely were analyzed separately. In addition, analyses were run with and without IQ as a covariate, in order to rule out the possibility that IQ had a differential effect on growth in both groups. All analyses were performed with software package SPSS version 16.0. Correction for multiple comparisons according to the False Discovery Rate (FDR) controlling procedure was applied to the analyses with a q-value setting of 0.05 (Benjamini and Hochberg 1995).

\section{Results}

Results were similar with and without inclusion of premature children, and so data for the entire group are presented. Although IQ was strongly related to several growth parameters and differed between the groups (proportionally more children with ASD had a lower IQ compared with PC children, see Table 1), inclusion of IQ as a covariate in the analyses did not change the main findings (results with IQ as covariate are available upon request from the first author), and so results are presented without IQ as covariate. Lastly, the growth of the children with autism was not different from that of the children with PDD-NOS (head circumference $[F(1,125)=0.01, p=.91]$; head circumference minus height $[F(1,125)=0.14, p=.71]$; height $[F(1,125)=0.02, p=.89]$; weight $[F(1,125)=$ $0.27, p=.60]$; BMI $[F(1,125)=0.65, p=.42])$, and so data are reported for the entire ASD group.

\section{ASD versus PC}

No structural group differences were found between ASD and PC children for any of the growth parameters, as indicated by non-significant main effects of group. In addition, the growth curves ran parallel for three of five growth parameters, as indicated by non-significant interaction effects between growth curve and group. Only two minor interaction effects emerged for head circumference and height, in which the PC children showed a slightly steeper growth curve compared with the ASD children. Thus, the two groups were more similar than dissimilar (see Table 2 and Fig. 1-5). In contrast, for all parameters, a deviation was found from a straight line through zero, suggesting that both groups tended not to grow as the population norm. Therefore, $t$-tests were performed comparing the $z$-scores of both groups separately to the population mean of zero. After correction for multiple testing, the following significant results emerged, which were largely comparable for both groups, except for head circumference in relation to height very early in development. $T$-tests revealed that only the ASD children had an increased head circumference relative to their height at the age of 1 month $(z=0.42, p<.001)$ and 2 months $(z=0.25, p=.006)$. Both groups had a decreased height at birth (ASD: $z=-0.40, p=.001$; PC: $z=-0.67, p=$ .002 ) and 1 month of age (ASD: $z=-0.50, p<.001$; PC: $z=-0.48, p=.005)$, and a decreased weight very early in development (ASD: birth $z=-0.34, p=.01 ; 1$ month $z=-0.35, p=.001$; PC: 1 month $z=-0.38, p=.005$ ). Similarly, BMI findings were largely comparable for both 
Table 1 Participants characteristics

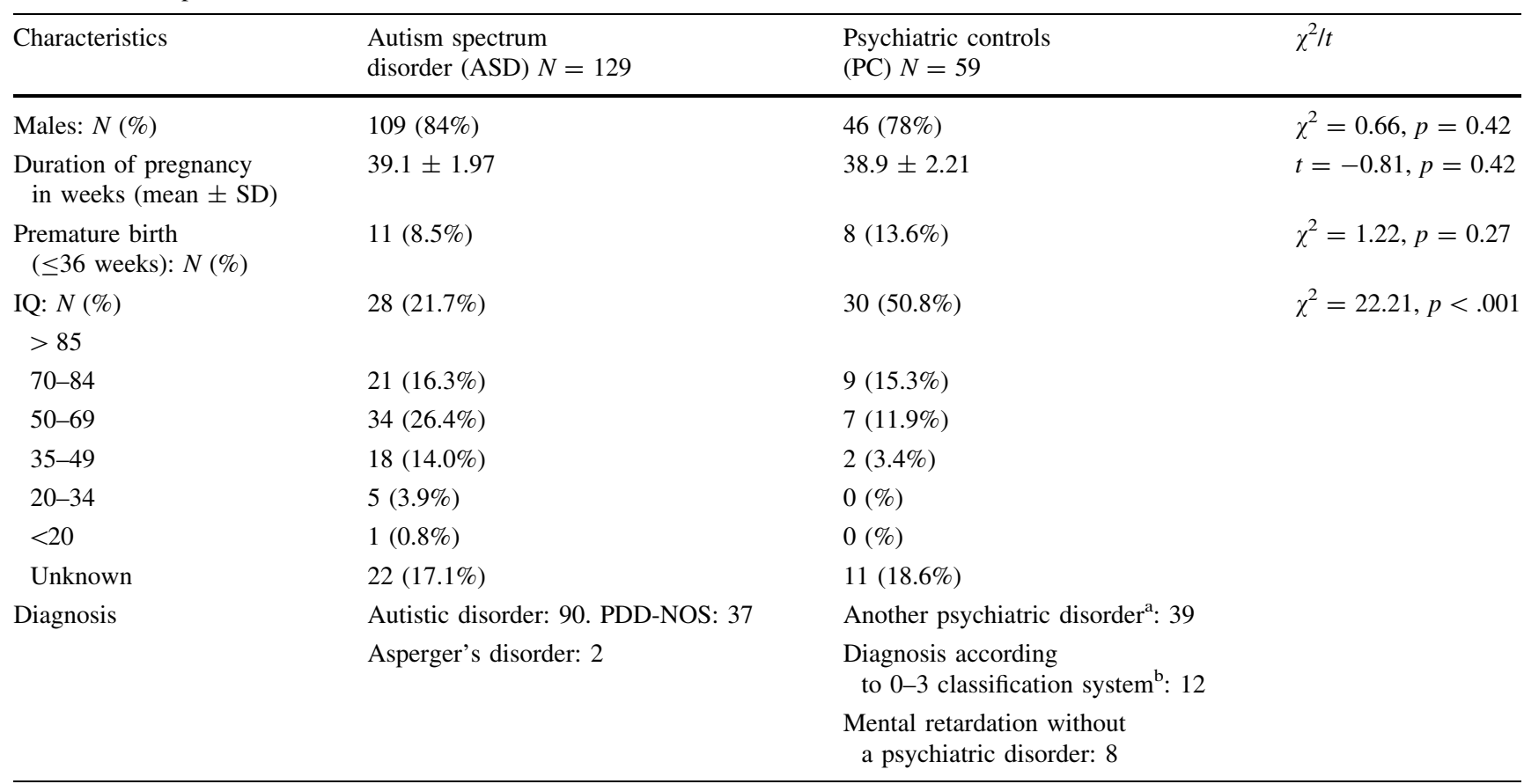

${ }^{a}$ Communication disorder $(N=16)$, Attention-deficit/hyperactivity disorder $(N=9)$, oppositional defiant disorder $(N=7)$, anxiety disorder $(N=3)$, affective disorder $(N=2)$, reactive attachment disorder $(N=2)$

${ }^{b}$ Diagnosis according to Diagnostic Classification of Mental Health and Developmental Disorders of Infancy and Early Childhood (Zero to Three 2005), such as regulation problems

Table 2 Results of the repeated measures ANOVAs comparing ASD children $(N=129)$ with PC children $(N=59)$ with respect to several growth parameters

\begin{tabular}{|c|c|c|c|c|c|c|c|}
\hline \multirow[t]{2}{*}{ Growth parameter } & \multicolumn{2}{|c|}{ Group } & \multicolumn{2}{|c|}{ Group $x$ growth curve } & \multicolumn{3}{|c|}{ Growth curve } \\
\hline & $F$ & $p$ & $F$ & $p$ & $F$ & $p$ & Pattern growth curve \\
\hline Head circumference & 1.20 & .27 & 2.75 & .01 & 3.64 & .001 & $\begin{array}{l}\text { Cubic: } F=9.99, p=.002 \\
\text { Linear: } F=4.04, p=.046\end{array}$ \\
\hline Head circumference minus height & 1.87 & .17 & 1.33 & .24 & 34.24 & $<.001$ & $\begin{array}{l}\text { Linear: } F=74.50, p<.001 \\
\text { Quadratic: } F=12.73, p<.001\end{array}$ \\
\hline Height & 0.04 & .85 & 2.26 & .02 & 38.14 & $<.001$ & $\begin{array}{l}\text { Linear: } F=66.58, p<.001 \\
\text { Quadratic: } F=15.85, p<.001 \\
\text { Cubic: } F=4.56, p=.03\end{array}$ \\
\hline Weight & 0.01 & .97 & 0.18 & .99 & 9.81 & $<.001$ & $\begin{array}{c}\text { Quadratic: } F=19.09, p<.001 \\
\text { Linear: } F=7.21, p=.008\end{array}$ \\
\hline Body mass index (BMI) & 0.03 & .86 & 0.39 & .93 & 16.53 & $<.001$ & $\begin{array}{l}\text { Linear: } F=30.53, p<.001 \\
\text { Quadratic: } F=7.33, p=.007 \\
\text { Cubic: } F=5.12, p=.03\end{array}$ \\
\hline
\end{tabular}

Findings printed in bold are significant after correction for multiple testing

groups. BMI was increased at birth in both groups (ASD: $z=0.46, p<.001$; PC: $z=0.52, p=.009)$, but decreased later in development (ASD 5-6 months: $z=-0.22, p=$ .02 ; $14-19$ months: $z=-0.24, p=.005$; PC 7-9 months: $z=-0.32, p=.01 ; 14-19$ months: $z=-0.33, p=.02$ ). Raw data is presented in Table 3.

\section{Abnormal Growth Acceleration}

Results are presented in Table 4. Neither group showed abnormal acceleration of head circumference or weight, but both groups showed a significant abnormal acceleration of height between birth and 14-19 months of age. Thus in 


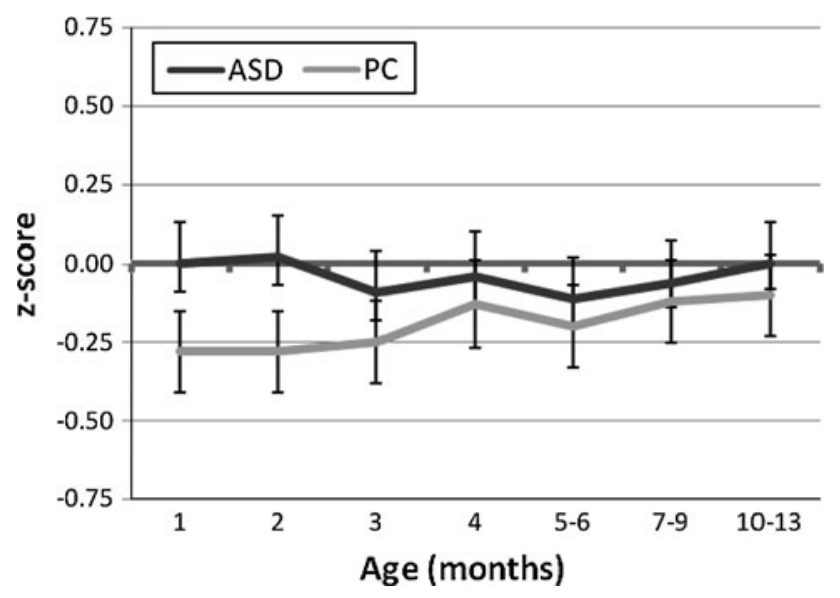

Fig. 1 Head circumference. Growth curves ( $z$-scores \pm 1 standard error) of autism spectrum disorder $(A S D)$ children compared to psychiatric control $(P C)$ children

both groups there was a significant abnormal deceleration of head circumference in relation to height and BMI.

\section{Microcephaly and Macrocephaly}

There were no differences between the ASD and PC groups regarding the proportion of children with microcephaly or macrocephaly (see Table 5).

\section{Discussion}

The aims of the current study were to examine whether abnormal early growth is (a) predominantly related to ASD or is generally found in children with psychiatric disorders (psychiatric controls [PC]), and (b) specific to the brain or generalized to the whole body. The first aim may shed light on whether growth parameters could be viable ASD-specific endophenotypes and whether abnormal growth is more broadly associated with developmental pathology.

Results revealed that, overall, the growth of ASD and PC children was more similar than dissimilar: repeated measure ANOVAs did not detect structural group differences in growth parameters, nor in group by growth curve interactions suggestive of significant long-term group differences in growth. This raises doubts about the use of abnormal growth as a marker of ASD pathology, because abnormal growth was not specific to ASD (Skuse 2001). Our findings suggest that growth abnormalities are a nonspecific expression of the biological abnormalities found in association with many psychiatric disorders. However, the one previous study that also examined this issue using longitudinal growth data did find differences in growth between ASD and children with developmental disabilities.
Webb et al. (2007) found that children with ASD had a larger head circumference at 1-7 months and were taller at 1-4 months than children with developmental disabilities. At 10 months, the children with ASD were generally larger than the children with developmental disabilities, having a larger head, height, and weight. The discrepancy between our results and those of Webb et al. may be explained by differences in the nature of the psychiatric control groups. Our psychiatric control group mainly consisted of children with psychiatric disorders (such as communication disorder, ADHD, ODD, anxiety disorder, affective disorder, reactive attachment disorder) or disturbances related to early development (such as regulation problems), whereas the few children in the developmental disabilities group $(N=8)$ of Webb et al. were selected on the basis of not passing certain developmental milestones (i.e., exhibiting problems in at least 3 of 5 of the following areas: verbal abilities, nonverbal abilities, nonverbal problem solving, motor milestones and adaptive behavior), rather than on the basis of psychiatric dysfunction. One other study that contrasted autistic children with ADHD children found no differences in head circumference at birth (Gillberg and de Souza 2002). Therefore, on the basis of our preliminary findings, differences in growth parameters appear to be a non-specific expressions of the biological abnormalities associated with psychiatric disorders.

However, even though the groups did not differ significantly overall, growth abnormalities might be helpful for creating more homogeneous subgroups of patients, to facilitate gene detection. The most robust finding from our and previous studies is the heterogeneity of growth trajectories. Some patients with ASD may have similar growth trajectories and form an etiologically more homogeneous subgroup. In addition, time-specific $t$-tests did reveal some subtle differences between the groups. The most apparent difference was that the children with ASD had an increased head circumference relative to height up to 2 months of age, which was not observed in the PC children. This initial disproportionately large head in comparison to height in the first 2 months of life may be important to our understanding of the unique etiological factors underlying ASD and not other psychiatric disorders.

In both groups of children, growth parameters deviated from a straight line through zero (representing a growth curve completely in conformity with population means), but the shape of the growth curve was not the same for all the parameters. Head circumference showed a predominantly cubic growth curve, whereby the head circumference tended to be smaller than the norm at birth and normalized later. This is consistent with some previous studies documenting a decreased head circumference at birth in ASD (Courchesne et al. 2003; Dissanayake et al. 2006; Mraz et al. 2007). Nevertheless, the head circumference did not 


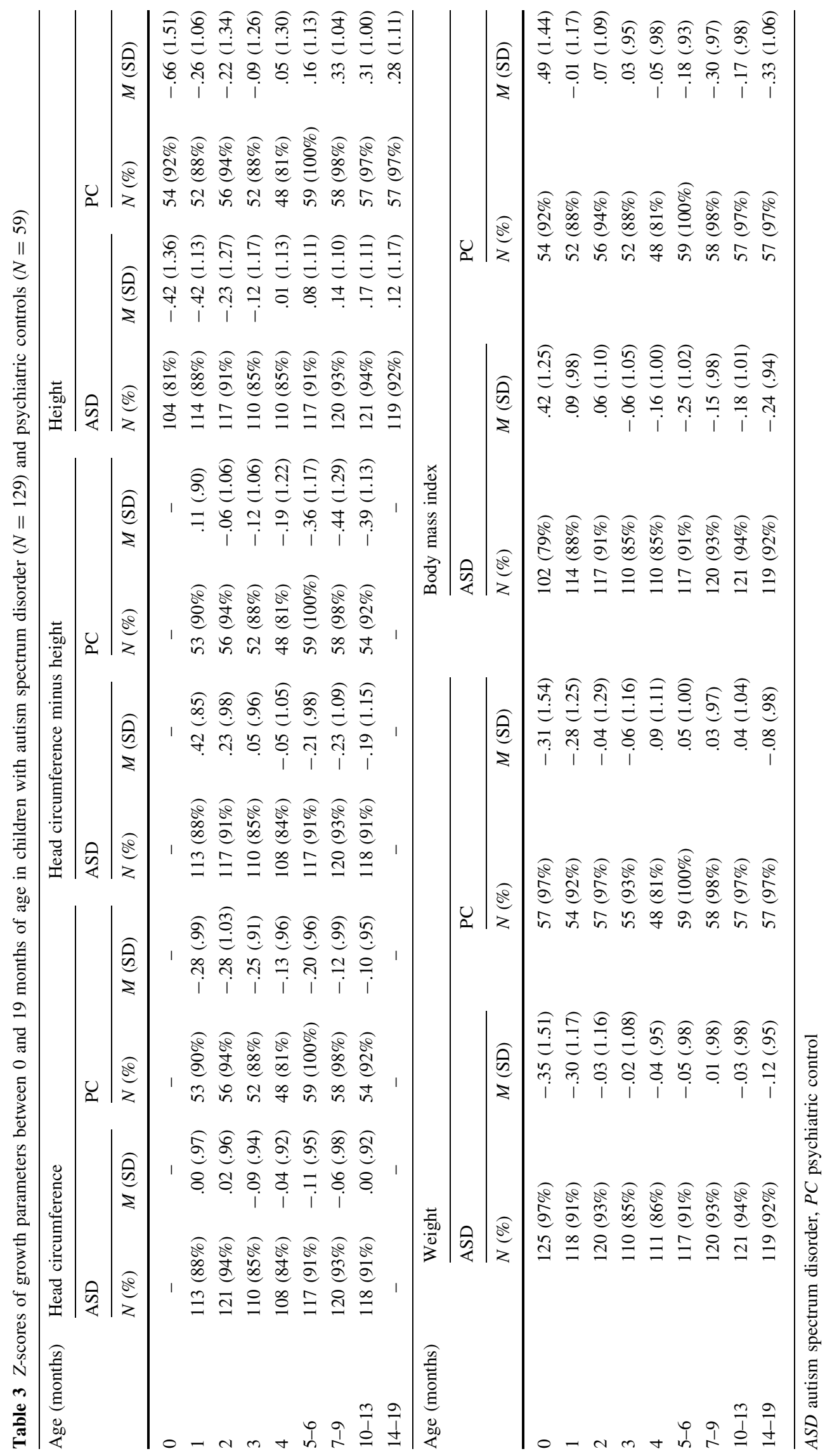


Table 4 Abnormal growth acceleration in children with ASD and PC defined as $\geq+1.8$ SD increase in head circumference from 1 to 10-13 months of age and as $\geq+2$ SD increase in height, weight or body mass index from 0 to 14-19 months of age

\begin{tabular}{|c|c|c|c|c|c|c|c|c|}
\hline & \multicolumn{2}{|c|}{$\operatorname{ASD}(N=129)$} & \multicolumn{2}{|l|}{$\mathrm{PC}(N=59)$} & \multicolumn{2}{|c|}{ ASD versus $\mathrm{PC}$} & \multirow{2}{*}{$\begin{array}{l}\text { ASD versus } \\
\text { norm population } \\
t\end{array}$} & \multirow{2}{*}{$\begin{array}{l}\text { PC versus } \\
\text { norm population } \\
t\end{array}$} \\
\hline & $M^{\mathrm{a}}(\mathrm{SD})$ & $N(\%)$ & $M^{\mathrm{a}}(\mathrm{SD})$ & $N(\%)$ & $t$ & $\chi^{2}$ & & \\
\hline Head circumference & $.08(.84)$ & $3(2.3 \%)$ & $.30(.94)$ & $3(5.1 \%)$ & 1.63 & 1.00 & 1.04 & 2.44 \\
\hline Head circumference minus height & $-.57(.88)$ & $1(0.8 \%)$ & $-.47(.85)$ & $0(0 \%)$ & 0.75 & .46 & -7.38 & -4.27 \\
\hline Height & $.50(1.36)$ & $15(11.6 \%)$ & $.96(1.68)$ & $12(20.3 \%)$ & 2.00 & 2.50 & 4.18 & 4.40 \\
\hline Weight & $.20(1.50)$ & $12(9.3 \%)$ & $.31(1.47)$ & $7(11.9 \%)$ & 0.49 & .29 & 1.51 & 1.63 \\
\hline Body mass index & $-.71(1.44)$ & $6(4.7 \%)$ & $-.85(1.63)$ & $2(3.4 \%)$ & -0.61 & .16 & $-\mathbf{5 . 5 7}$ & -4.02 \\
\hline
\end{tabular}

$A S D$ autism spectrum disorder, $P C$ psychiatric control

Bold significant after correction for multiple testing

${ }^{a}$ Mean difference between z-score at 10-13 months of age (head circumference and head circumference minus height) or 14-19 months of age (height, weight and body mass index) and z-score at 1 month of age (head circumference and head circumference minus height) or at birth (height, weight and body mass index)

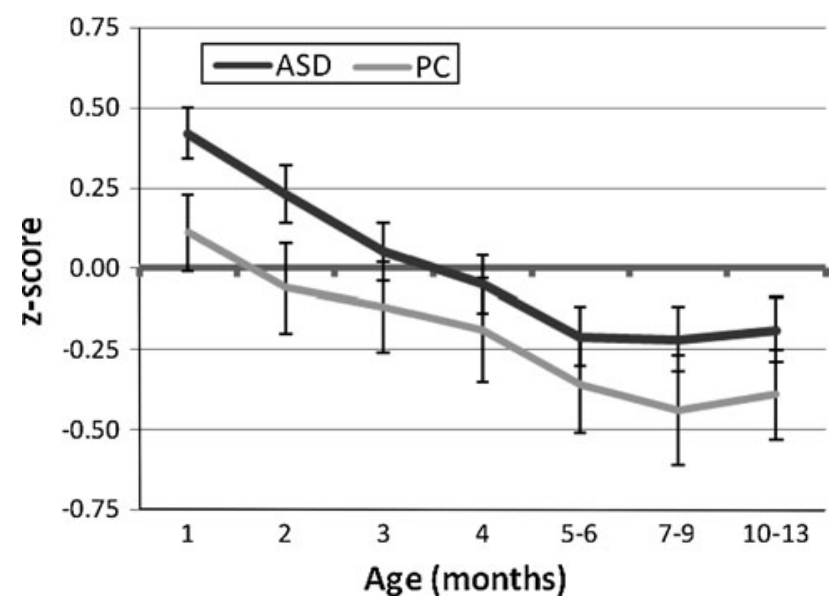

Fig. 2 Head circumference minus height parameter. Growth curves ( $z$-scores \pm 1 standard error) of autism spectrum disorder $(A S D)$ children compared to psychiatric control $(P C)$ children

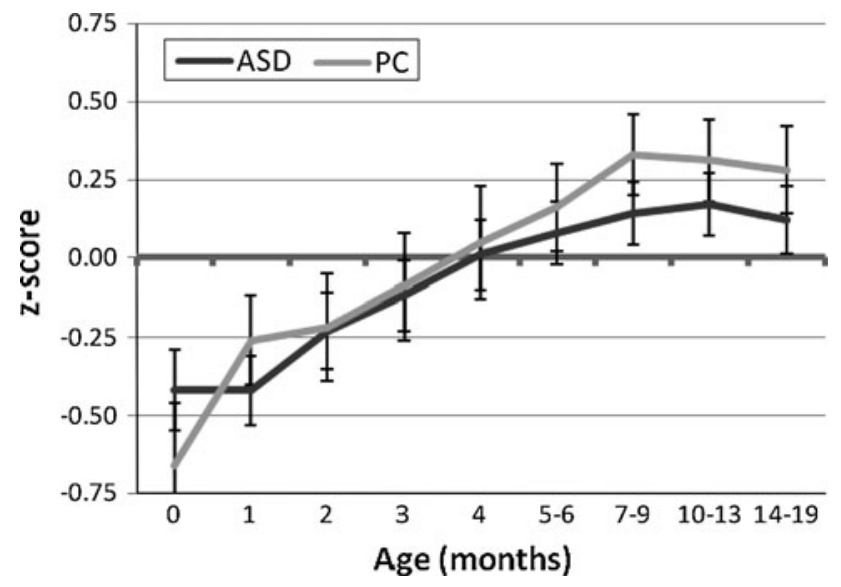

Fig. 3 Height. Growth curves ( $z$-scores \pm 1 standard error) of autism spectrum disorder $(A S D)$ children compared to psychiatric control $(P C)$ children

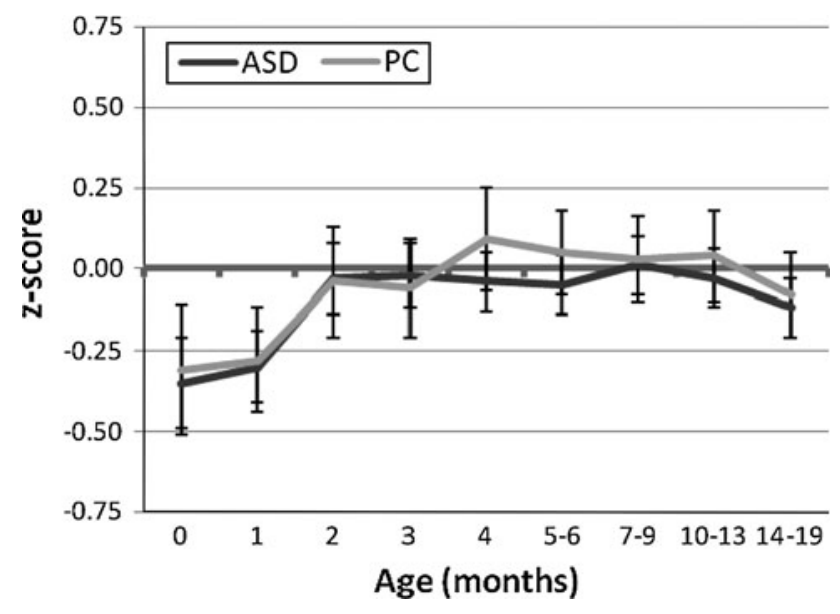

Fig. 4 Weight. Growth curves ( $z$-scores \pm 1 standard error) of autism spectrum disorder $(A S D)$ children compared to psychiatric control $(P C)$ children

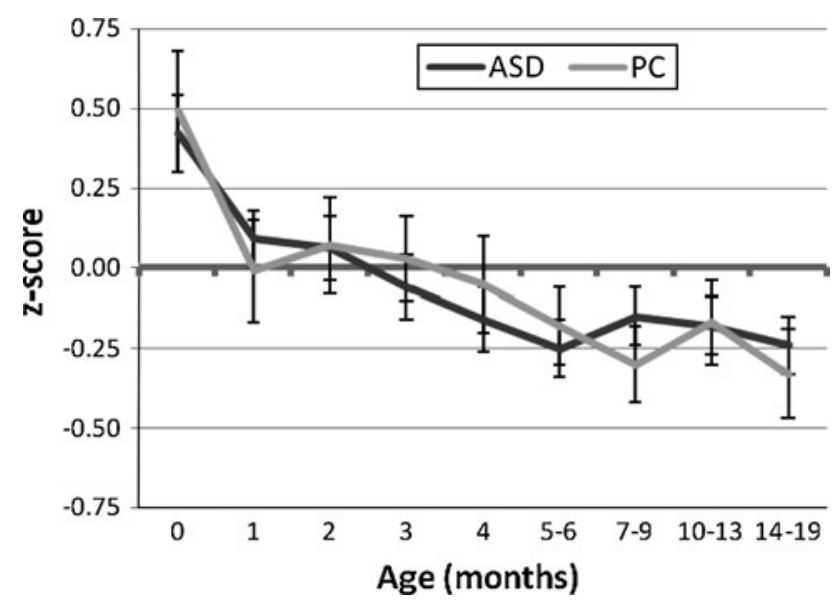

Fig. 5 Body mass index. Growth curves ( $z$-scores \pm 1 standard error) of autism spectrum disorder $(A S D)$ children compared to psychiatric control $(P C)$ children 
Table 5 Number of children with microcephaly ( $\leq 2$ SD below population mean) or macrocephaly ( $\geq 2$ SD above population mean)

\begin{tabular}{|c|c|c|c|c|c|}
\hline \multirow[t]{2}{*}{ Age (months) } & \multicolumn{2}{|l|}{ Microcephaly } & \multicolumn{2}{|l|}{ Macrocephaly } & \multirow[t]{2}{*}{$\chi^{2}, p$} \\
\hline & $\begin{array}{l}\text { ASD }(N=129) \\
N(\%)\end{array}$ & $\begin{array}{l}\mathrm{PC}(N=59) \\
N(\%)\end{array}$ & $\begin{array}{l}\text { ASD }(N=129) \\
N(\%)\end{array}$ & $\begin{array}{l}\mathrm{PC}(N=59) \\
N(\%)\end{array}$ & \\
\hline 1 & $5(3.9 \%)$ & $4(6.8 \%)$ & $2(1.6 \%)$ & $0(0 \%)$ & $1.64, .44$ \\
\hline 2 & $4(3.1 \%)$ & $3(5.1 \%)$ & $1(0.8 \%)$ & $1(1.7 \%)$ & $0.79, .68$ \\
\hline 3 & $3(2.3 \%)$ & $1(1.7 \%)$ & $2(1.6 \%)$ & $1(1.7 \%)$ & $0.08, .96$ \\
\hline 4 & $3(2.3 \%)$ & $2(3.4 \%)$ & $1(0.8 \%)$ & $0(0 \%)$ & $0.63, .73$ \\
\hline $5-6$ & $2(1.6 \%)$ & $2(3.4 \%)$ & $2(1.6 \%)$ & $0(0 \%)$ & $1.56, .46$ \\
\hline $7-9$ & $2(1.6 \%)$ & $0(0 \%)$ & $2(1.6 \%)$ & $1(1.7 \%)$ & $0.93, .63$ \\
\hline $10-13$ & $2(1.6 \%)$ & $0(0 \%)$ & $6(4.7 \%)$ & $1(1.7 \%)$ & $1.95, .38$ \\
\hline
\end{tabular}

$A S D$ autism spectrum disorder, $P C$ psychiatric control

show an abnormal accelerated growth and the proportion of children with macrocephaly was not higher or lower than normal, in contrast to some previous ASD studies (Courchesne et al. 2003; Dawson et al. 2007; Dementieva et al. 2005; Gillberg and de Souza 2002; Lainhart et al. 1997). Our findings suggest that the pattern of head circumference development was in the main normal. However, the growth curve of head circumference in relation to height indicated that ASD children had a larger head relative to their length at birth, which then showed an abnormal growth deceleration, resulting in a head circumference that was small relative to height over time, as shown previously (van Daalen et al. 2007). Notwithstanding these findings, the differences were very small $(-0.25 z$ score $)$ and given that population growth curves are constructed from thousands of individuals, it is difficult to say whether these findings are typical. With respect to general body growth, the children with ASD tended to be smaller than normal at birth (possibly due to a higher than normal prevalence of prenatal and perinatal risk factors in our ASD sample [unpublished data]), yet showed an abnormal growth acceleration, becoming taller than normal children, consistent with earlier reports of abnormally increased height (van Daalen et al. 2007; Fukumoto et al. 2008; Torrey et al. 2004). At the same time, their weight did not increase proportionally, making these children on average taller and thinner than normal children. Thus both ASD and PC children grew differently from the population norms. In general, these children were smaller at birth, but were significantly taller at about 1.5 years. Head circumference and body weight did not match this 'growth spurt'-both parameters lagged somewhat behind the growth in height, making these children as a group somewhat taller, thinner, and with proportionally smaller heads than age- and sex-matched peers. The etiology of these growth abnormalities may be genetic, environmental (such as maternal substance abuse, poor nutrition, intrauterine infections, etc.), or a combination of both. While we do not want to suggest that the growth abnormalities of children with ASD or other psychiatric disorders are solely genetically based, we believe that shared genetic factors influence psychiatric functioning and growth in these children.

Our results should be interpreted in the context of the limitations of this pilot study. Most importantly, the PC group was very heterogeneous. A more homogeneous control group, such as children with mental retardation of a specific type, might have revealed greater differences in growth. We repeated the analyses, comparing children with communication disorders $(N=16)$ and ADHD/ODD $(N=16)$ with the children with ASD. Except for a main group difference in head circumference between the ASD and $\mathrm{ADHD} / \mathrm{ODD}$ group $(p=.05)$, the head circumference of the children with ASD was normal, whereas that of the children with ADHD/ODD was smaller than normal, no other main effects or interaction effects emerged between the groups (results available upon request). Thus, given that children with ADHD are also known to show early growth abnormalities (although often in relation to risk factors such as prematurity, smoking, and hypertension), our findings suggest that growth parameters are related to psychiatric dysfunctioning in general. Secondly, it is likely that a proportion of our ASD sample suffered from one or more comorbid psychiatric conditions, such as ADHD. However, this is probably also true of other samples of young children with ASD. Our PC group had been thoroughly screened and found to be negative for ASD. The crucial difference between the two groups is the presence/ absence of ASD, which makes comparison of the two groups viable. A related limitation is the young age at which our subjects were diagnosed, so that autism, particularly Asperger's disorder and high functioning autism, could not be excluded with certainty in the children in the PC group and could only be confirmed in the ASD group as they became older. We are in the process of investigating this. Fourthly, the IQ differences between the ASD and PC groups could have influenced the results. However, even though IQ was strongly related to several growth 
parameters, including IQ as a covariate in the analyses did not influence the lack of group differences or group by growth curve interactions. Socioeconomic status, which could influence growth, was not taken in consideration. However, differences in socioeconomic status are not large in the Netherlands, and Lainhart et al. (2006) did not find socioeconomic status to be associated with head size in autistic children. Lastly, it would have been valuable to have included a third group of control children to exclude the possibility of specific cohort effects, but this would not have influenced the findings of similar abnormal growth in ASD and PC children.

In conclusion, our study showed that growth abnormalities appear to be a non-specific expression of biological abnormalities found in several psychiatric disorders. The growth of both ASD and PC children differed from population norms. Generally, these children were smaller at birth, but after a period of abnormal growth acceleration, they were significantly taller than normative samples of children at about 1.5 years. Head circumference and body weight did not match this 'growth spurt' in height, so that these children as a group were somewhat taller, thinner, and with proportionally smaller heads than age- and sexmatched peers. Although head circumference, weight, and height did not distinguish between the ASD group and the PC group, the growth trajectories of the two groups were heterogeneous.

We aim to replicate and extend these findings by examining whether head circumference differs between ASD probands, genetically related siblings with ADHD but without ASD, and a group of genetically unrelated children with ADHD but no ASD relatives. This may help answer the question whether growth abnormalities are predominantly associated with ASD (in which case the first group will show on average more abnormalities than the other two groups), or associated with more general heritable/ familial risk factors in ASD families (in which case the ASD probands and ADHD-affected siblings will show on average more abnormalities than the genetically unrelated ADHD children), or associated with psychiatric problems in general (in which case the three groups will not differ substantially from each other, but will differ from population norms).

Open Access This article is distributed under the terms of the Creative Commons Attribution Noncommercial License which permits any noncommercial use, distribution, and reproduction in any medium, provided the original author(s) and source are credited.

\section{References}

Amaral, D. G., Schumann, C. M., \& Nordahl, C. W. (2008). Neuroanatomy of autism. Trends in Neurosciences, 31, 137-145.
American Psychiatric Association. (2000). Diagnostic and statistical manual of mental disorders, 4th edn, text revision. Washington, DC: American Psychiatric Association.

Bartholomeusz, H. H., Courchesne, E., \& Karns, C. M. (2002). Relationship between head circumference and brain volume in healthy normal toddlers, children and adults. Neuropediatrics, 33, 239-241.

Benjamini, Y., \& Hochberg, Y. (1995). Controlling the false discovery rate: a practical and powerful approach to multiple testing. Journal of the Royal Statistical Society B, 5, 289-300.

Carmichael, C. M., \& McGue, M. (1995). A cross-sectional examination of height, weight, and body mass index in adult twins. Journal of Gerontology series A: Biological Sciences and Medical Sciences, 50, 237-244.

Carper, R. A., Moses, P., Tigue, Z. D., \& Courchesne, E. (2002). Cerebral lobes in autism: Early hyperplasia and abnormal age effects. NeuroImage, 16, 1038-1051.

Chawarska, K., Klin, A., Paul, R., \& Volkmar, F. (2007). Autism spectrum disorder in the second year: Stability and change in syndrome expression. Journal of Child Psychology and Psychiatry, 48, 128-138.

Courchesne, E. (1997). Brainstem, cerebellar and limbic neuroanatomical abnormalities in autism. Current Opinion in Neurobiology, 7, 269-278.

Courchesne, E., Carper, R., \& Akshoomoff, N. (2003). Evidence of brain overgrowth in the first year of life in autism. Journal of the American Medical Association, 290, 337-344.

Courchesne, E., Karns, C. M., Davis, H. R., Ziccardi, R., Carper, R. A., Tique, Z. D., et al. (2001). Unusual brain growth patterns in early life in patients with autistic disorder: An MRI study. Neurology, 57, 245-254.

Courchesne, E., Redcay, E., \& Kennedy, D. P. (2004). The autistic brain: birth through adulthood. Current Opinion in Neurology, 17, 489-496.

Dawson, G., Munson, J., Webb, S. J., Nalty, T., Abbott, R., \& Toth, K. (2007). Rate of head growth decelerates and symptoms worsen in the second year of life in autism. Biological Psychiatry, 61, 458-464.

Dementieva, Y. A., Vance, D. D., Donnelly, S. L., Elston, L. A., Wolpert, C. M., Ravan, S. A., et al. (2005). Accelerated head growth in early development of individuals with autism. Pediatric Neurology, 32, 102-108.

Dissanayake, C., Bui, Q. M., Huggins, R., \& Loesch, D. Z. (2006). Growth in stature and head circumference in high-functioning autism and Asperger disorder during the first 3 years of life. Development and Psychopathology, 18, 381-393.

Elder, L. M., Dawson, G., Toth, K., Fein, D., \& Munson, J. (2008). Head circumference as an early predictor of autism symptoms in younger siblings of children with autism spectrum disorder. Journal of Autism and Developmental Disorders, 38, 11041111.

Fredriks, A. M., van Buuren, S., Brugmeijer, R. J., Meulmeester, J. F., Beuker, R. J., Brugman, E., et al. (2000). Continuing positive secular growth change in the Netherlands 1955-1997. Pediatric Research, 47, 316-323.

Fukumoto, A., Hashimoto, T., Hiromichi, I., Nishimura, M., Tsuda, Y., Miyazaki, M., et al. (2008). Growth of head circumference in autistic infants during the first year of life. Journal of Autism and Developmental Disorders, 38, 411-418.

Gillberg, C., \& de Souza, L. (2002). Head circumference in autism, Asperger's syndrome and ADHD: a comparative study. Developmental Medicine and Child Neurology, 44, 296-300.

Gottesman, I. I., \& Gould, T. D. (2003). The endophenotype concept in psychiatry: Etymology and strategic intentions. American Journal of Psychiatry, 160, 636-645. 
Hazlett, H. C., Poe, M., Gerig, G., Smith, R. G., Provenzale, J., Ross, A., et al. (2005). Magnetic resonance imaging and head circumference study of brain size in autism: Birth through age 2 years. Archives of General Psychiatry, 62, 1366-1376.

Lainhart, J. E., Bigler, E. D., Bocian, M., Coon, H., Dinh, E., Dawson, G., et al. (2006). Head circumference and height in autism: A study by the collaborative program of excellence in autism. American Journal of Medical Genetics, 140A, 2257-2274.

Lainhart, J. E., Piven, J., Wzorek, M., Landa, R., Santangelo, S. L., Coon, H., et al. (1997). Macrocephaly in children and adults with autism. Journal of the American Academy of Child \& Adolescent Psychiatry, 36, 282-290.

Lawler, C. P., Croen, L. A., Grether, J. K., \& Van de Water, J. (2004). Identifying environmental contributions to autism: Provocative clues and false leads. Mental Retardation and Developmental Disabilities Research Reviews, 10, 292-302.

Lord, C., Rutter, M., DiLavore, P., \& Risi, S. (2001). Autism diagnostic observation schedule (ADOS) manual. Los Angeles, CA: Western Psychological Services.

Losh, M., Sullivan, P. F., Trembath, D., \& Piven, J. (2008). Current developments in the genetics of autism: From phenome to genome. Journal of Neuropathology and Experimental Neurology, 67, 829-837.

Maes, H. H. M., Neale, M. C., \& Eaves, L. J. (1997). Genetic and environmental factors in relative body weight and human adiposity. Behavior Genetics, 27, 325-351.

Mills, J. L., Hediger, M. L., Molloy, C. A., Chrousos, G. P., ManningCourtney, P., Yu, K. F., et al. (2007). Elevated levels of growthrelated hormones in autism and autism spectrum disorder. Clinical Endocrinology, 67, 230-237.

Mraz, K. D., Green, J., Dumont-Mathieu, T., Makin, S., \& Fein, D. (2007). Correlates of head circumference growth in infants later diagnosed with autism spectrum disorders. Journal of Child Neurology, 22, 700-713.

Mullen, E. (1995). Mullen scales of early learning (AGS ed.). Circle Pines, MN: American Guidance Service.

Oosterling, I. J., Wensing, M., Swinkels, S. H., van der Gaag, R. J., Visser, J. C., Woudenberg, T., et al. (2010). Advancing early detection of autism spectrum disorder by applying a two-stage screening approach. epub ahead of print: Journal of Child Psychology and Psychiatry.

Redcay, E., \& Courchesne, E. (2005). When brain enlarged in autism? A meta-analysis of all brain size reports. Biological Psychiatry, $58,1-9$.

Rutter, M., Le Couteur, A., \& Lord, C. (2003). ADI-R: Autism diagnostic interview-Revised. Los Angeles, CA: Western Psychological Services.

Schlichting, J. E. P. T., van Eldik, M. C. M., Lutje Spelberg, H. C., van der Meulen, S. J., \& van der Meulen, B. F. (1995). Schlichting test voor taalproductie. Lisse: Swets Test Publishers.

Schopler, E., Reichler, R. J., Bashford, A., Lansing, M. D., \& Marcus, L. M. (1990). The psychoeducational profile revised (PEP-R). Austin: Pro-Ed.
Silventoinen, K., Kaprio, J., Lahelma, E., \& Koskenvuo, M. (2000). Relative effect of genetic and environmental factors on body height: differences across birth cohorts among Finnish men and women. American Journal of Public Health, 90, 627-630.

Skuse, D. H. (2001). Endophenotypes and child psychiatry. British Journal of Psychiatry, 178, 395-396.

Sparks, B. F., Friedman, S. D., Shaw, D. W., Aylward, E. H., Echelard, D., Artru, A. A., et al. (2002). Brain structural abnormalities in young children with autism spectrum disorder. Neurology, 59, 184-192.

Swinkels, S. H., Dietz, C., van Daalen, E., Kerkhof, I. H., van Engeland, H., \& Buitelaar, J. K. (2006). Screening for autistic spectrum in children aged 14 to 15 months. I: The development of the early screening of autistic traits questionnaire (ESAT). Journal of Autism and Developmental Disorders, 36, 723-732.

Tabachnick, B. G., \& Fidell, L. S. (2001). Using multivariate statistics (4th ed.). Needham Heights: Allyn and Bacon.

Torrey, E. F., Dhavale, D., Lawlor, J. P., \& Yolken, R. H. (2004). Autism and head circumference in the first year of life. Biological Psychiatry, 56, 892-894.

van Daalen, E., Swinkels, S. H. N., Dietz, C., van Engeland, H., \& Buitelaar, J. K. (2007). Body length and head growth in the first year of the life in autism. Pediatric Neurology, 37, 324-330.

van Eldik, M. C. M., Schlichting, J. E. P. T., Lutje Spelberg, H. C., van der Meulen, B. F., \& van der Meulen, S. J. (1995). Reynell test voor taalbegrip. Amsterdam: Harcourt Test Publishers.

Webb, S. J., Nalty, T., Munson, J., Brock, C., Abbott, R., \& Dawson, G. (2007). Rate of head circumference growth as a function of autism diagnosis and history of autistic regression. Journal of Child Neurology, 22, 1182-1190.

Whitely, P., Dodou, K., Todd, L., \& Shattock, P. (2004). Body mass index of children from United Kingdom diagnosed with pervasive developmental disorders. Pediatrics International, $46,531-533$.

Wiggins, L. D., Baio, J., \& Rice, C. (2006). Examination of the time between first evaluation and first autism spectrum diagnosis in a population-based sample. Journal of Development and Behavioral Pediatrics, 27, 79-87.

Xiong, N., Ji, C., Li, Y., He, Z., Bo, H., \& Zhao, Y. (2007). The physical status of children with autism in China. Research in Developmental Disabilities, 30, 70-76.

Zero to Three. (2005). Diagnostic classification of mental health and developmental disorders of infancy and early childhood: Revised edition (DC:0-3R). Washington, DC: ZERO TO THREE Press.

Zink, I., \& Lembrecht, D. (2000). Nederlandstalige nonspeech test. Leusden: Acco.

Zwaigenbaum, L., Bryson, S., Lord, C., Rogers, S., Carter, A., Carver, L., et al. (2009). Clinical assessment and management of toddlers with suspected autism spectrum disorder: Insights from studies of high-risk infants. Pediatrics, 123, 1383-1391. 